\title{
ESCENARIOS CLIMÁTICOS Y RECURSOS HÍDRICOS SOBRE LA REGIÓN DE MURCIA A MEDIADOS DEL SIGLO XXI*
}

\author{
J. Quereda Sala, E. Montón Chiva, J. Escrig Barberá \\ Laboratorio de Clima \\ Universitat Jaume I
}

\begin{abstract}
RESUMEN
El objetivo del presente estudio ha sido el de analizar y proyectar los impactos que un cambio climático podría tener sobre el territorio de la Comunidad de Murcia. Un objetivo de gran trascendencia a tenor de las actuales predicciones que los modelos desarrollados establecen sobre esta región. A tenor de los análisis efectuados, nuestras conclusiones sobre las consecuencias de un posible cambio climático sobre la Región de Murcia no descartan la posibilidad de un sostenimiento climático dentro de la variabilidad natural de nuestro clima. No obstante, con las incertidumbres expresadas y ante la gran sensibilidad que los recursos hídricos muestran frente a las variaciones climáticas, hemos realizado una proyección sobre las tendencias termopluviométricas más severas observadas durante el siglo XX.
\end{abstract}

Palabras clave: cambio climático, predicción, Murcia.

\begin{abstract}
The present work examines and forecasts the impacts of climatic change over the Comunidad de Murcia territory. It's a goal of a great importance because of the scenarios that the climatic models get for this region. Our conclusions don't reject a future climatic stability around the natural variability. Nevertheless, with these uncertainties and by the important sensibility the water resources show in the presence of climatic changes, we have forecasted the temperature and rainfall trends following the worst scenarios obtained along the XX century.
\end{abstract}

Key-words: climatic change, forecast, Murcia.

* El presente trabajo constituye una síntesis de la proyección climática realizada en el proyecto «Estrategias frente al cambio climático» (Gobierno autonómico de la Comunidad de Murcia), co-dirigido con Garrigues MedioAmbiente. 


\section{Introducción}

La búsqueda de un modelo climático para el siglo XXI que integre todas las anomalías observadas, se ha convertido en uno de los mayores retos científicos actuales. No es para menos por cuanto que el mayor impacto ambiental previsto para los próximos cincuenta o cien años viene constituido por el cambio climático global básicamente atribuido a la actividad humana. En este orden de conocimientos, las bases científicas del último informe IPCC (Panel Intergubernamental para el Cambio Climático), Climate Change 2001 de las Naciones Unidas, basadas en los modelos del Hadley Centre, establecen que el incremento global de la temperatura durante el siglo $\mathrm{XX}$ ha sido de $0.6{ }^{\circ} \mathrm{C} \pm 0.2{ }^{\circ} \mathrm{C}$. Un incremento térmico que para el rango de escenarios desarrollado en el IPCC, Special Report on Emision Scenarios (SRES), va a suponer un calentamiento global entre $1.4{ }^{\circ} \mathrm{C}$ y $5.8^{\circ} \mathrm{C}$ hacia el año 2100 (figura 1). Incrementos energéticos sobre los que los modelos desarrollados establecen un gradual aumento del nivel del mar entre 0.09 y 0.88 metros para el mismo año.

Estos mismos modelos proyectados por el Hadley Centre, para un escenario de un incremento anual del $1 \%$ en la concentración atmosférica del $\mathrm{CO}_{2}$, predicen que nuestra región mediterránea será la más vulnerable de Europa a los efectos del cambio climático. Vulnerabilidad basada en que simultáneamente al aumento térmico de 2 ó $3{ }^{\circ} \mathrm{C}$, se produciría una reducción de recursos hídricos. El valor de la misma, en el modelo de tercera generación (HadCM3) podría alcanzar hasta un $33 \%$ de las actuales magnitudes de escorrentía mediterráneas, ya de por sí escasas con valores medios anuales de $75 \mathrm{~mm}$ en las tierras valencianas y tan sólo $50 \mathrm{~mm}$ en la Región de Murcia. Esta escorrentía total equivale a unos recursos hídricos naturales de $990 \mathrm{Hm}^{3}$ año sobre la Cuenca del Segura y, consecuentemen-

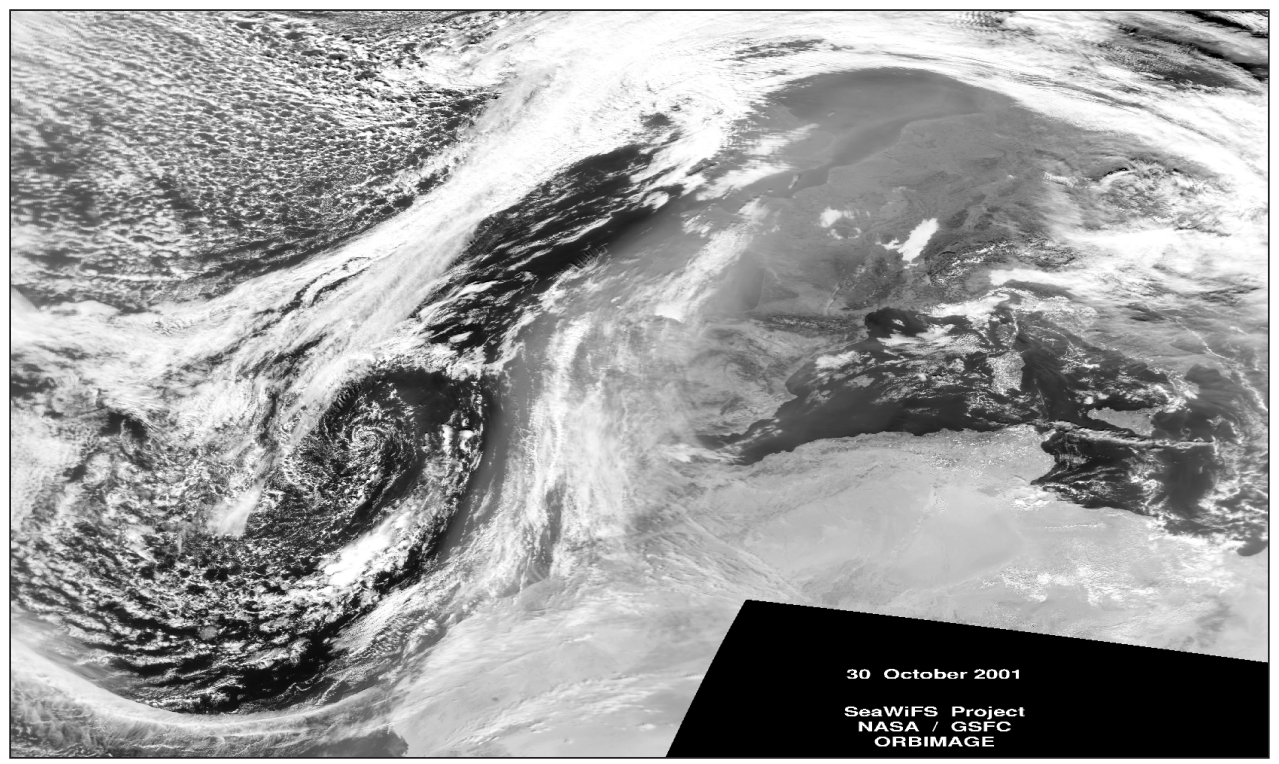

FIGURA 1. Espectacular transporte de polvo africano sobre toda la Europa Occidental. La frecuencia de estos aerosoles podría monitorizar un eventual desplazamiento de la zona desértica sobre ámbitos ibéricos. 
te, la reducción en un tercio de sus escorrentías, tan sólo dejaría unos recursos aproximados de $620 \mathrm{Hm}^{3}$ año con aumento de su déficit actual que ya alcanza los $450 \mathrm{Hm}^{3}$ año. Un escenario previsto en la reciente planificación hidráulica en España (figura 2).

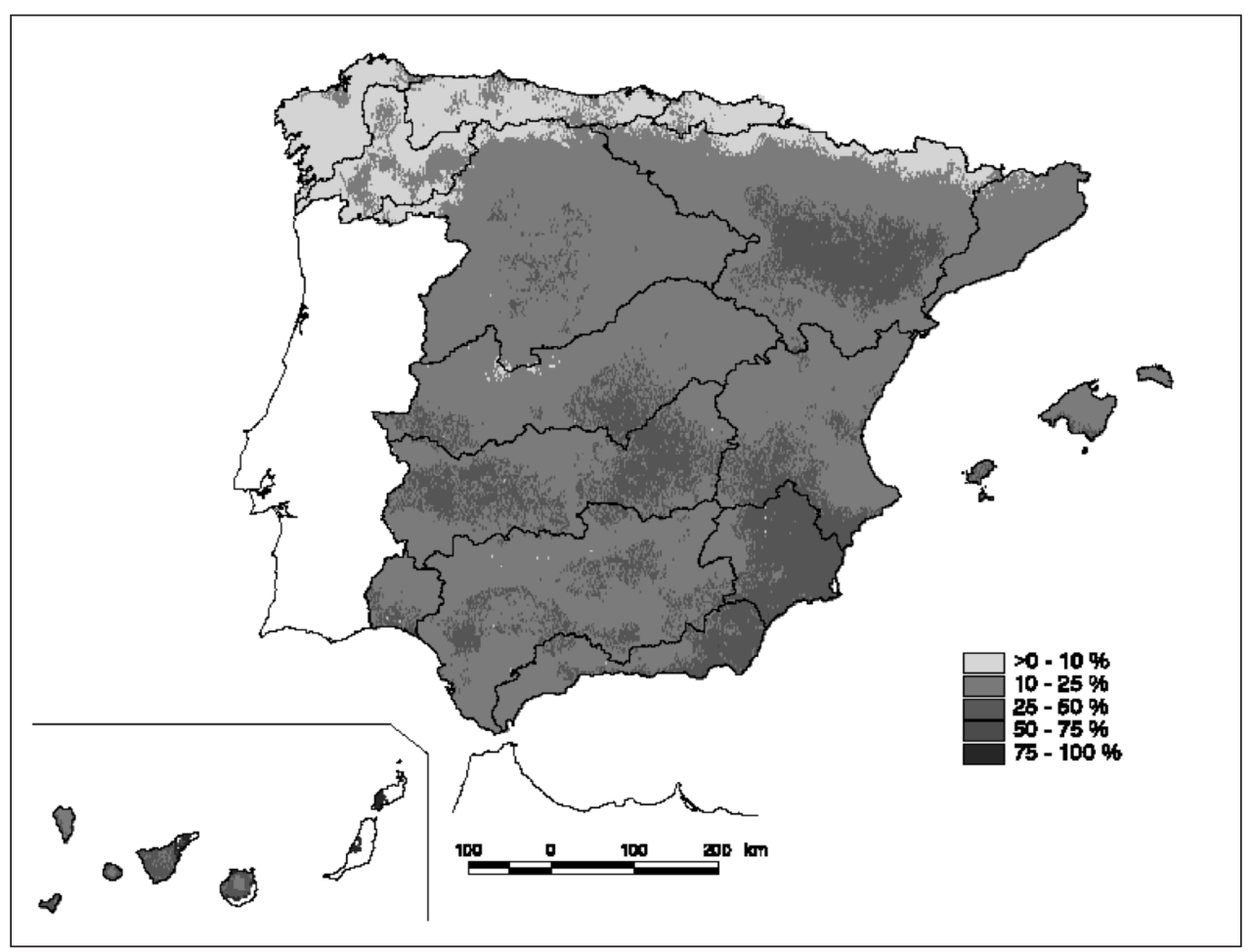

Figura 2. Libro Blanco del Agua. Escenario n 2. Impacto sobre los recursos hídricos a través de la reducción de la escorrentía. La Región de Murcia podría sufrir una grave reducción de hasta el 50 \% en sus escorrentías.

\section{Los modelos climáticos globales y sus incertidumbres}

Consecuentemente, y dentro de ese escenario global o planetario, la cuestión es: ¿Qué clima cabe esperar a nuestra región mediterránea a lo largo de la primera mitad del siglo XXI? Y, más precisamente, ¿Cuál va a ser el escenario climático sobre las tierras de la Región de Murcia? Una cuestión a la que el desarrollo de los modelos globales de la circulación (CGMs) ha venido aportando aproximaciones interesantes aunque muy inseguras. La principal debilidad de los mismos reside en nuestra insuficiente comprensión del Sistema Climático y de sus ingredientes claves: agua y energía.

Las transferencias de energía entre la atmósfera y los océanos son un factor determinante en el equilibrio y las variaciones del clima. Extendiéndose sobre las tres cuartas partes de la superficie del globo, pudiendo almacenar, para una duración más o menos prolongada, considerables cantidades de energía, capaces de transportar esa energía sobre millares de kilómetros y asegurando en su superficie lo esencial del mecanismo de evapo- 
ración con transformación del calor sensible en calor latente, los océanos juegan un papel central y complejo dentro de los procesos energéticos globales y regionales. Y es precisamente en este dominio de las interaciones atmósfera-mar donde se suscitan las mayores incertidumbres y muy especialmente en el papel del vapor de agua, el principal componente energético de la atmósfera y a la vez el más intenso de los GEIs.

\section{Una proyección de los posibles escenarios climáticos de la Región de Murcia en el siglo XXI}

Sin embargo, a pesar de la formidable aportación de los modelos de cambio climático, las simulaciones del clima futuro realizadas por ellos, sobre sistemas cerrados océanoatmósfera de la Circulación General, resultan todavía muy inciertas. Realmente es muy difícil prevenir cual va a ser el impacto de ese efecto e incluso su misma existencia. En este orden de conocimientos consideramos que los análisis de la evolución climática regional, pueden contribuir eficazmente a configurar un escenario de la evolución climática futura, especialmente en ámbitos espaciales complejos y reducidos.

A este respecto, y al objeto de configurar el posible escenario climático de la Región de Murcia durante el siglo XXI, hemos procedido inicialmente a determinar el comportamiento y las tendencias climáticas registradas en la densa red de observatorios meteorológicos regionales. Un conocimiento que simultáneamente ha permitido precisar dos objetivos. El primero ha sido el de verificar si las tendencias climáticas regionales en temperaturas y precipitaciones, muestran una evolución secular acorde con los escenarios previstos por los modelos cerrados atmósfera-océano de Cambio Climático Global. El segundo objetivo ha sido el de proporcionar a nuestro diseño de escenario climático futuro las tendencias latentes que el clima ha venido mostrando en los finales del siglo XX y que, sin duda, podrían estar anticipando las condiciones venideras. Ésta será la proyección del escenario climático futuro desarrollada en los epígrafes 4 y 5.

\subsection{Resultados del análisis: la evolución secular de las temperaturas en la Región de Murcia}

El análisis de la evolución térmica regional ha sido efectuado sobre una red que comprende tres estaciones de primer orden y treinta de segundo orden (figura 3). Esta red corresponde básicamente a la Región de Murcia, si bien para dotar de una mayor precisión a los análisis y cartografía se han incluido alguno de los observatorios de la Provincia de Alicante que climáticamente proyectan la vertiente marítima de la propia región de Murcia. Los datos de base (INM, Instituto Nacional de Meteorología) han sido las medias mensuales de las temperaturas medias mínimas y máximas. Series cuya calidad ha sido testeada y normalizada en función del método de homogeneización de Alexandersson y Moberg (1997).

La evolución de la temperatura media anual muestra una apreciable tendencia de calentamiento climático. Esta tendencia media regional ha alcanzado valores de $0.5^{\circ} \mathrm{C}$ a lo largo de la segunda mitad del siglo XX. Sin embargo, tanto los diferentes valores entre observatorios (figura 4), como la diferencia de comportamiento entre las temperaturas máximas y las mínimas sugieren una heterogeneidad de calentamiento y, consecuentemente, algunas incertidumbres y reflexiones (figura 5 ).

A este respecto, y tras el complejo y laborioso proceso de homogeneización y corrección de heterogeneidades, podemos estimar que una parte de esa elevación de la temperatura, 0.5 ${ }^{\circ} \mathrm{C}$ sobre la segunda mitad del siglo XX, podría resultar del efecto urbanización. Un efecto 


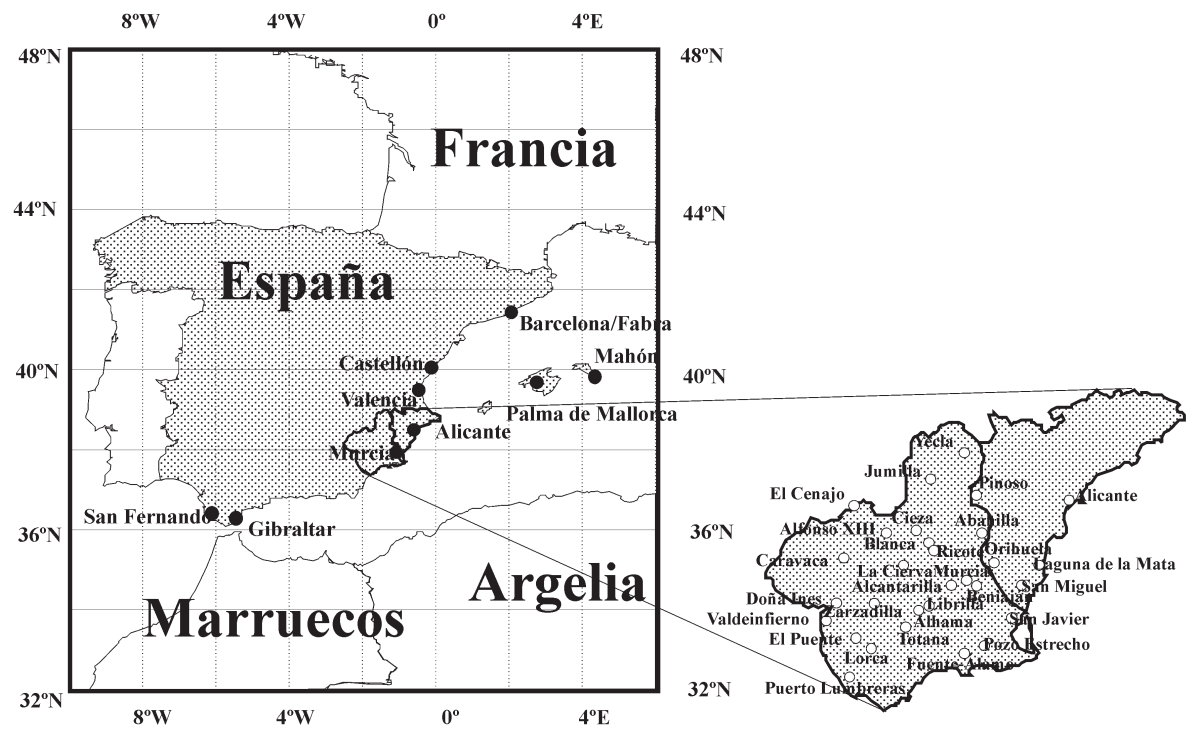

Figura 3. Mapa de los principales observatorios de la Región de Murcia y Provincia de Alicante utilizados en este estudio.

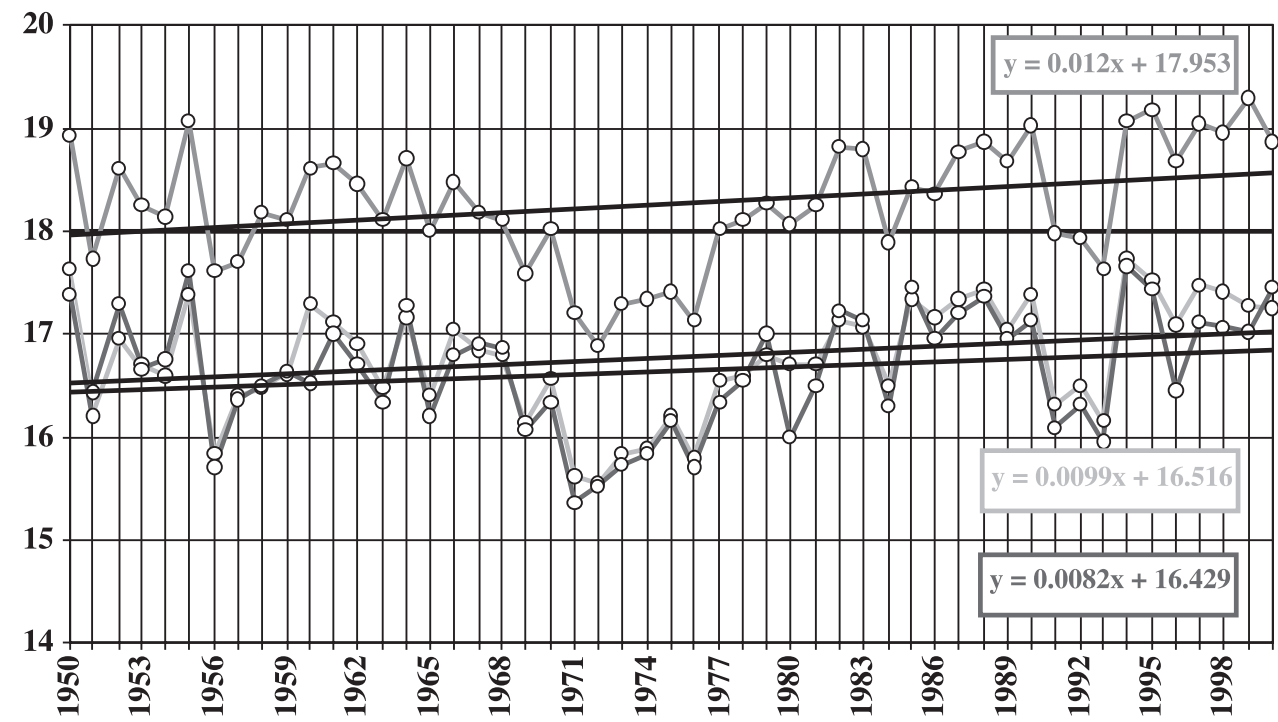

FIGURA 4. Evolución y tendencia de la temperatura media anual en los observatorios urbanos de Murcia, Elche y Alicante, así como en los restantes observatorios «más rurales» (De 80.000 a 20.000 y menores de 20.000 habitantes) Fuente: INM. Elaboración propia. 


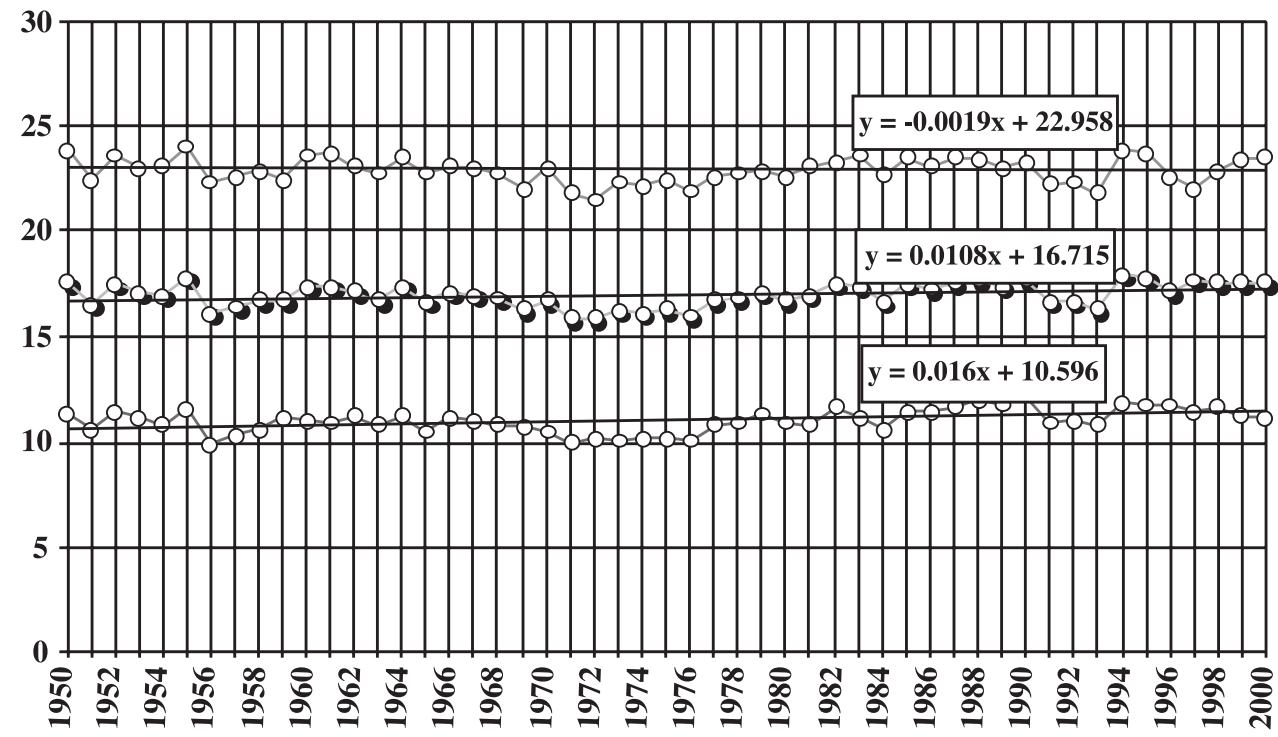

Figura 5. Evolución y tendencia de las temperaturas medias anual, máxima y mínima en los observatorios de la Región de Murcia y de la Provincia de Alicante. Fuente: INM. Elaboración propia.

que se manifiesta progresiva y acumulativamente ya que los observatorios han sido absorbidos paulatinamente por la expansión de las ciudades. El resultado de este proceso puede venir reflejado en el hecho de que toda la elevación térmica se ha venido produciendo exclusivamente en las temperaturas mínimas, con un valor de $0{ }^{\prime} 8^{\circ} \mathrm{C}$ a lo largo del periodo 1950-2000. La evolución de las temperaturas máximas, con -0 ' $2{ }^{\circ} \mathrm{C}$, igualmente durante la segunda mitad del siglo XX, apenas ha mostrado tendencia alguna y en todo caso ésta sería de enfriamiento.

La evolución térmica del observatorio de Murcia resulta paradigmática en este orden de conocimientos. La historia del observatorio meteorológico de Murcia ha proporcionado una buena experiencia sobre los procesos térmicos asociados al crecimiento de las ciudades. Experiencia que, en síntesis, ha permitido «substraer» la influencia de calor urbano que podría venir afectando a los termómetros del observatorio. El observatorio, creado en 1869 , se trasladó en 1954 desde el Instituto Alfonso X a la Universidad, donde permaneció catorce años hasta su nuevo traslado a Vistabella en 1967. En Vistabella permanece hasta 1984 año en que se traslada a la villa de Guadalupe, si bien el INM ha mantenido el observatorio de Vistabella de modo que las observaciones vienen siendo simultáneas. Los traslados iniciales, especialmente el de 1967 desde la Universidad, en el centro urbano, a Vistabella en el exterior, quedan reflejados en el gran retraso y amortiguamiento de los efectos urbanos sobre las temperaturas registradas (figura 6).

Cuando estos efectos, que ya se comenzaban a registrar, podrían haberse dejado sentir más intensamente el observatorio es desplazado (1967) hacia una periferia abierta junto al río Segura, Vistabella. En cualquier caso, la aportación urbana queda de manifiesto en la evolución seguida por las temperaturas mínimas en el observatorio de Vistabella y la crisis que experimentan con el traslado al nuevo observatorio situado en la localidad vecina de 


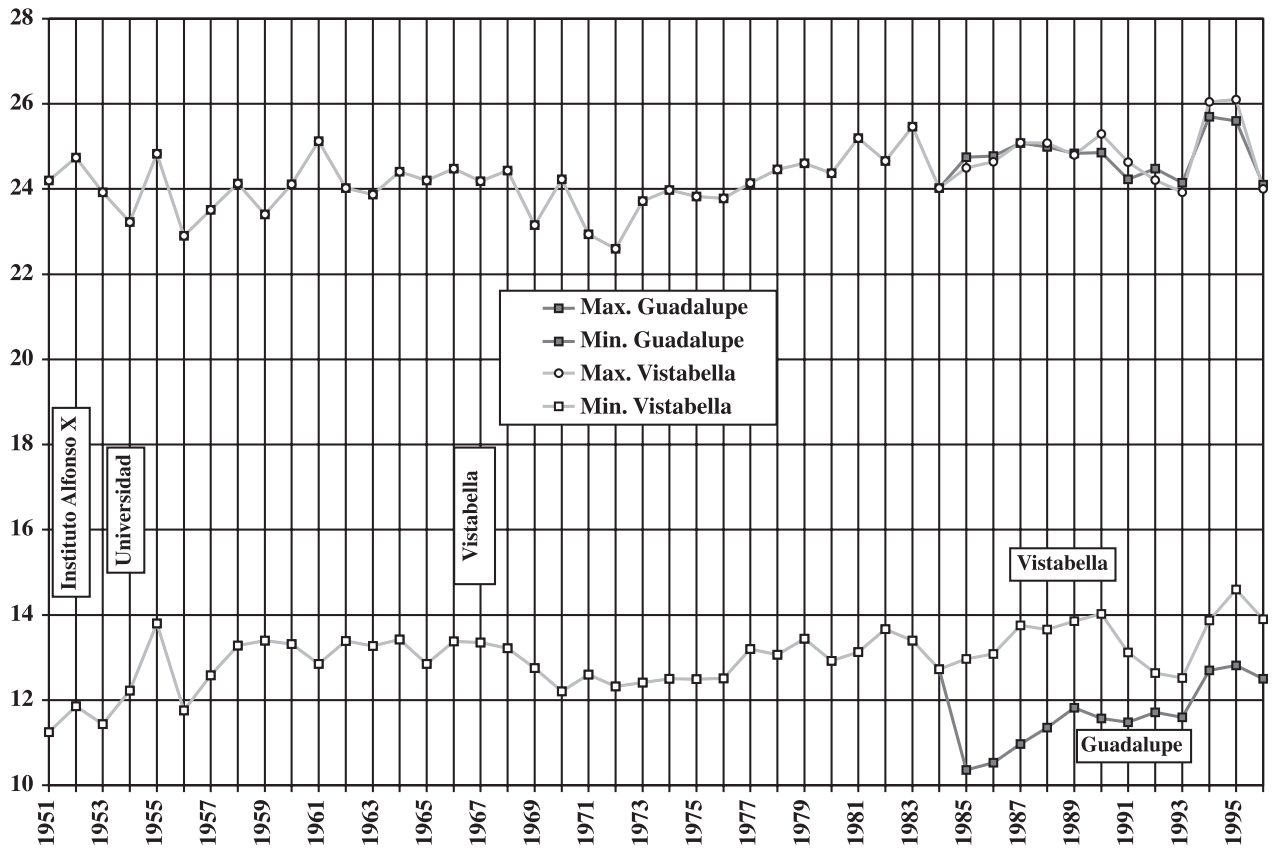

Figura 6. Evolución de las temperaturas máximas y mínimas del observatorio de Murcia en sus diversas ubicaciones. Fuente: INM. Elaboración propia.

Guadalupe. Una aportación que, insensible en las temperaturas máximas, se acusa bruscamente en el notable descenso de las temperaturas mínimas desde 1985, año del traslado a Guadalupe. Es además muy significativo que el mismo observatorio de Guadalupe esté registrando una elevación progresiva de las temperaturas mínimas en los últimos años. Un signo que igualmente viene a mostrar que ese $0.4{ }^{\circ} \mathrm{C}$ de aumento térmico «natural» estimado sobre el valor de tendencia de los observatorios «rurales» también podría estar afectado artificialmente.

\subsection{Resultados del análisis: la evolución de las precipitaciones en la Región de Murcia}

Con la misma metodología científica utilizada para el tratamiento de las temperaturas, el análisis de las series pluviométricas de la Comunidad de Murcia y de la Provincia de Alicante ha mostrado una tendencia hacia la reducción de las precipitaciones a lo largo de la segunda mitad del siglo XX. Esta reducción ha alcanzado un valor de trend de $0.65 \mathrm{~mm}$ anuales, es decir de $32 \mathrm{~mm}$ al año entre la década de 1950 y finales de siglo (figura 7).

No obstante esta tendencia negativa no se manifiesta más que durante la segunda mitad del siglo XX, ya que si la establecemos a partir de 1934 con los veinte observatorios que tienen series completas, el valor del trend resulta altamente positivo. Como consecuencia del mismo las precipitaciones habrían mostrado un incremento de $65 \mathrm{~mm}$ entre 1934 y 2000 (figura 8). 


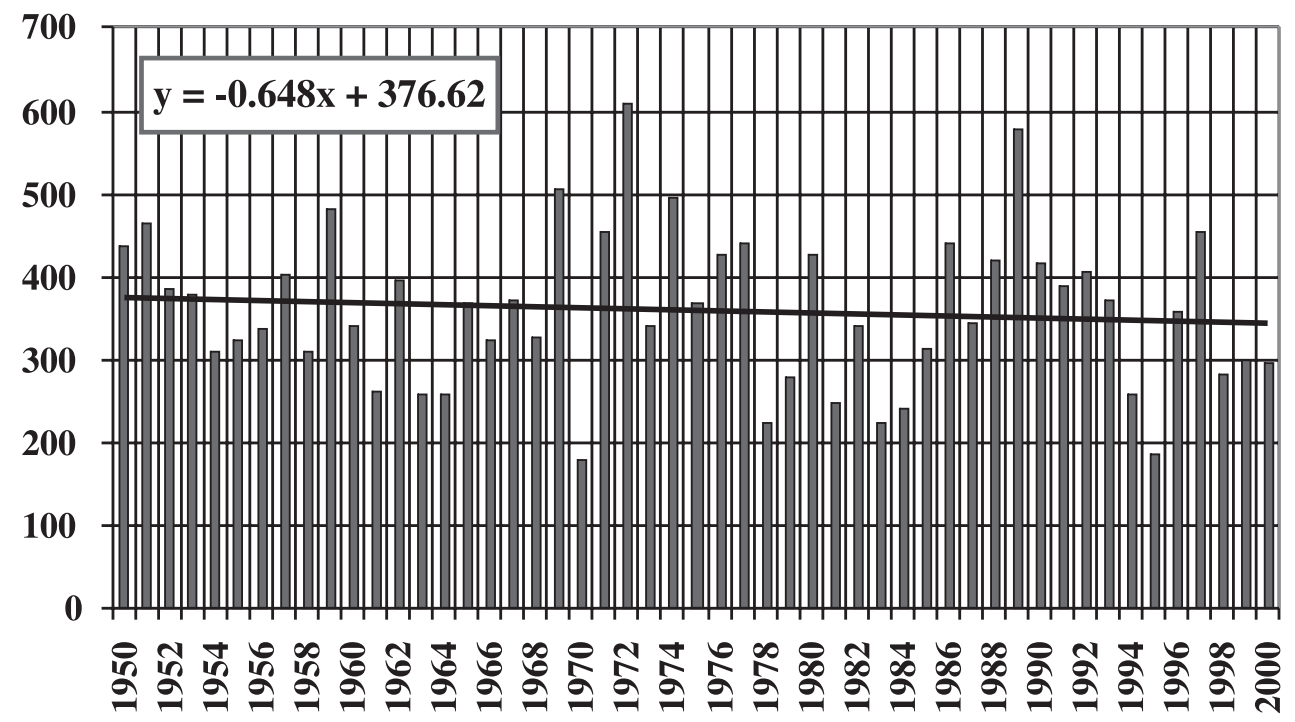

Figura 7. Evolución de las precipitaciones en la Región de Murcia a lo largo de la segunda mitad del siglo XX.

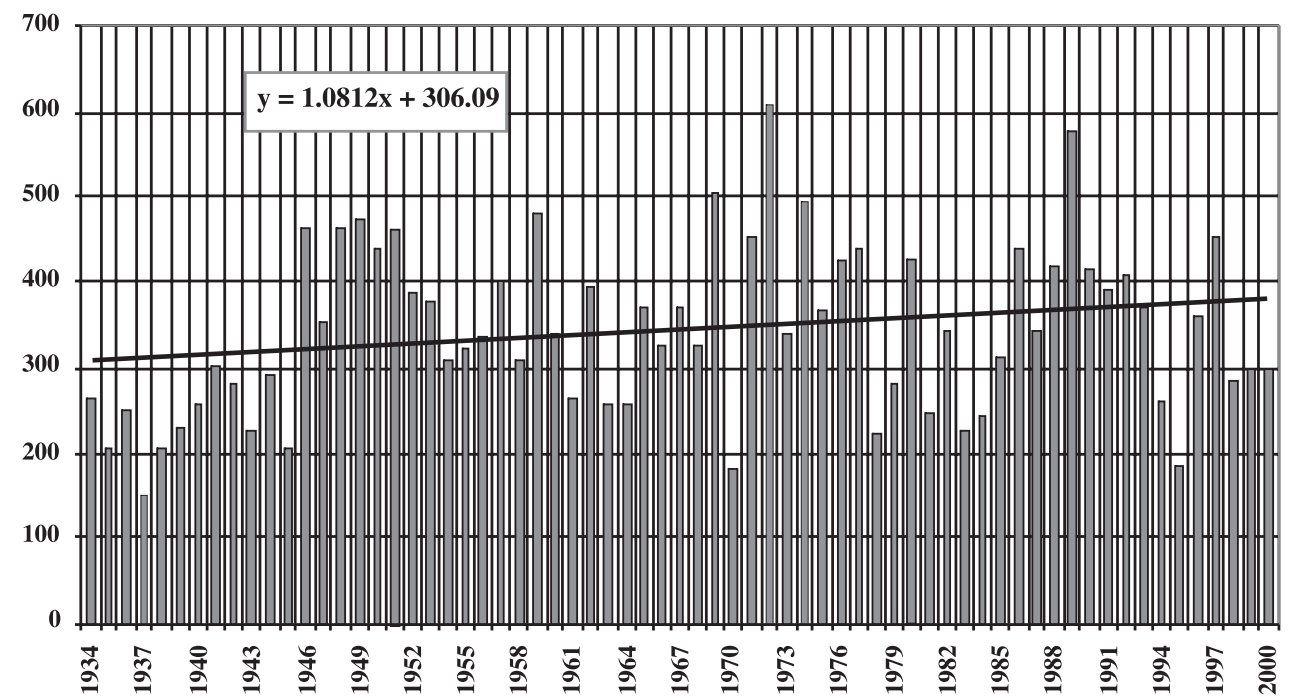

FIgURA 8. La evolución pluviométrica de la Región de Murcia muestra una tendencia netamente positiva entre los años 1934 a 2000. 


\section{La previsión del escenario climático sobre la Región de Murcia en el horizonte del año 2050: impactos sobre el ciclo hidrológico}

Como consecuencia de todos los análisis y procesos analizados, creemos que la mejor ponderación de los escenarios climáticos previsibles sobre la Región de Murcia en el horizonte del año 2050 puede venir encuadrada por tres umbrales:

I) No es posible descartar un primer escenario basado en la hipótesis de la sostenibilidad climática con sus variabilidades naturales. Una hipótesis justificada en la heterogeneidad de la evolución térmica y pluviométrica observada y carente de significación estadística.

II) Un segundo escenario, contemplaría un aumento de $0.5^{\circ} \mathrm{C}$ en la temperatura media anual y un sostenimiento de las actuales precipitaciones. Un escenario basado en las tendencias termopluviométricas seculares de todo el siglo XX.

III) Un tercer escenario, el de consecuencias más severas, contemplaría un aumento de hasta $0.5{ }^{\circ} \mathrm{C}$ en las temperaturas medias regionales y una reducción de un $5 \%$ en el volumen de sus precipitaciones medias. Este escenario corresponde a la proyección de las tendencias climáticas observadas durante la segunda mitad del siglo XX (1951-2000).

Sin duda que los dos últimos escenarios suscitan de inmediato una preocupación por el impacto que tales variaciones climáticas puedan tener sobre los recursos hídricos. En efecto, la modificación de las condiciones atmosféricas actuales induciría una modificación en el ciclo hidrológico natural y consecuentemente en los recursos hídricos naturales. En este sentido, las sequías pluviométricas podrían venir agravadas además por un crecimiento gradual de las evapotranspiraciones. Así pues, el concepto de ciclo hidrológico, síntesis de los procesos climáticos que se desarrollan sobre un territorio, es un concepto clave y como tal viene recibiendo una gran atención.

Esta atención está especialmente justificada en regiones tan deficitarias de agua como la Región de Murcia donde el papel de los recursos hídricos es el protagonista en la configuración territorial (Cerdá, 2003). En los momentos actuales, y ampliando el marco a toda la Cuenca del Segura como unidad hídrica independiente, la escorrentía se halla en valores inferiores a $50 \mathrm{~mm} / \mathrm{año}$, lo cual constituye la menor aportación específica del territorio español. Este valor es 20 veces inferior al de Galicia y 5 veces inferior a la media española (figura 9). De esta escorrentía total deriva la recarga natural o componente subterránea de los acuíferos. Una situación de escasez hídrica que todavía alcanza caracteres de mayor severidad sobre los $11.314 \mathrm{Km}^{2}$ de la Comunidad de Murcia y en la que tan sólo un $30 \%$ del territorio superaría los $10 \mathrm{~mm}$ de escorrentía media anual.

\section{La evaluación de los recursos hídricos sobre la Comunidad de Murcia}

En este orden de conocimientos, la evaluación de los recursos hídricos totales, superficiales y subterráneos, ha sido efectuada mediante la concepción del ciclo hidrológico. En este concepto, los recursos naturales propios son los que se generan a partir de la precipitación y que subsistentes a la evapotranspiración nutren y son aportados a través de las escorrentías superficiales directas y la recarga a los acuíferos. Lamentablemente el cálculo de este balance hidrológico en su régimen natural es una tarea que ofrece grandes dificultades y que técnicamente todavía no está bien resuelta. En efecto, los datos básicos para este conocimiento deberían estar basados en las medidas de las estaciones de aforos. Sabido es 


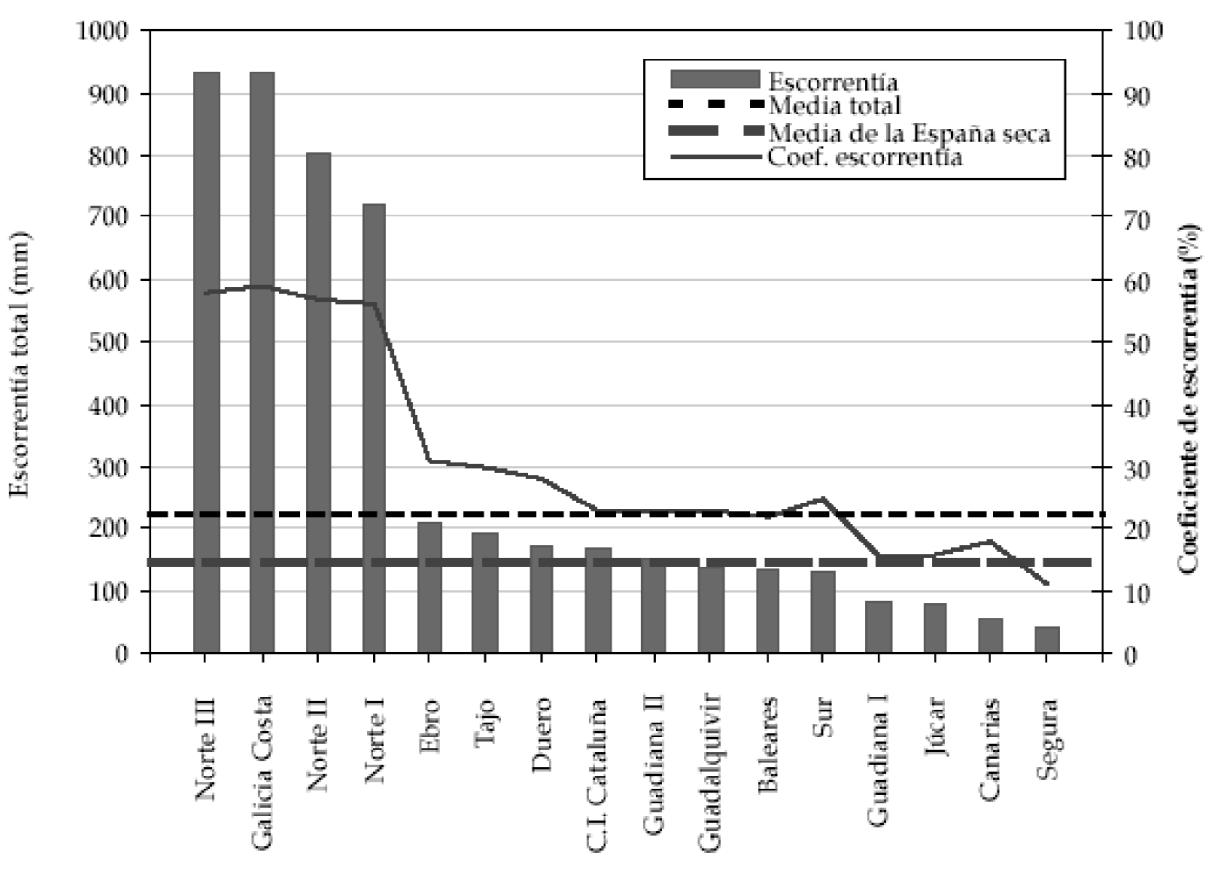

FIGURA 9. Escorrentías medias anuales $(\mathrm{mm})$ y coeficientes de escorrentía en régimen natural en las cuencas hidrográficas de España (Libro Blanco del Agua, MIMAM 2000).

que estos aforos miden simultáneamente tanto las aportaciones superficiales como también las subterráneas que en cada punto de la cuenca se incorporan al cauce procedentes de infiltraciones aguas arriba. Desgraciadamente los datos de las estaciones de aforos suelen medir regímenes afectados y no es fácil obtener una información suficiente sobre la evolución de los caudales detraídos o retornados de los ríos en sus diversos usos o afecciones. Todo ello determina que la restitución de los caudales en régimen natural sea una tarea de enormes dificultades (figura 10).

Frente a esta serie de dificultades que plantean los aforamientos y de las insuficientes redes de medida e información superficiales y subterráneas, la red meteorológica permite mejores resultados. En efecto, ante la estrecha dependencia que el ciclo hidrológico mantiene con los datos climáticos, el conocimiento de los principales valores hidrológicos del clima, nos permite proceder a su estimación. Esta estimación viene fundamentada en la dependencia que el valor de la escorrentía (E), o aportaciones totales (A), muestra con los valores de la precipitación (P) y con los de la evapotranspiración potencial (ETP). Este método permite alcanzar buenos resultados. Incluso por definición estos resultados pueden ser óptimos sobre la base de reunir dos condiciones. La primera es que la red meteorológica disponible no presente sesgos de localización que falseen la modelización posterior. La segunda, auténtico talón de Aquiles, es la medida de la evapotranspiración. Ambas condiciones han sido debidamente analizadas en nuestros cálculos tal y como se detallan en los apartados correspondientes. 


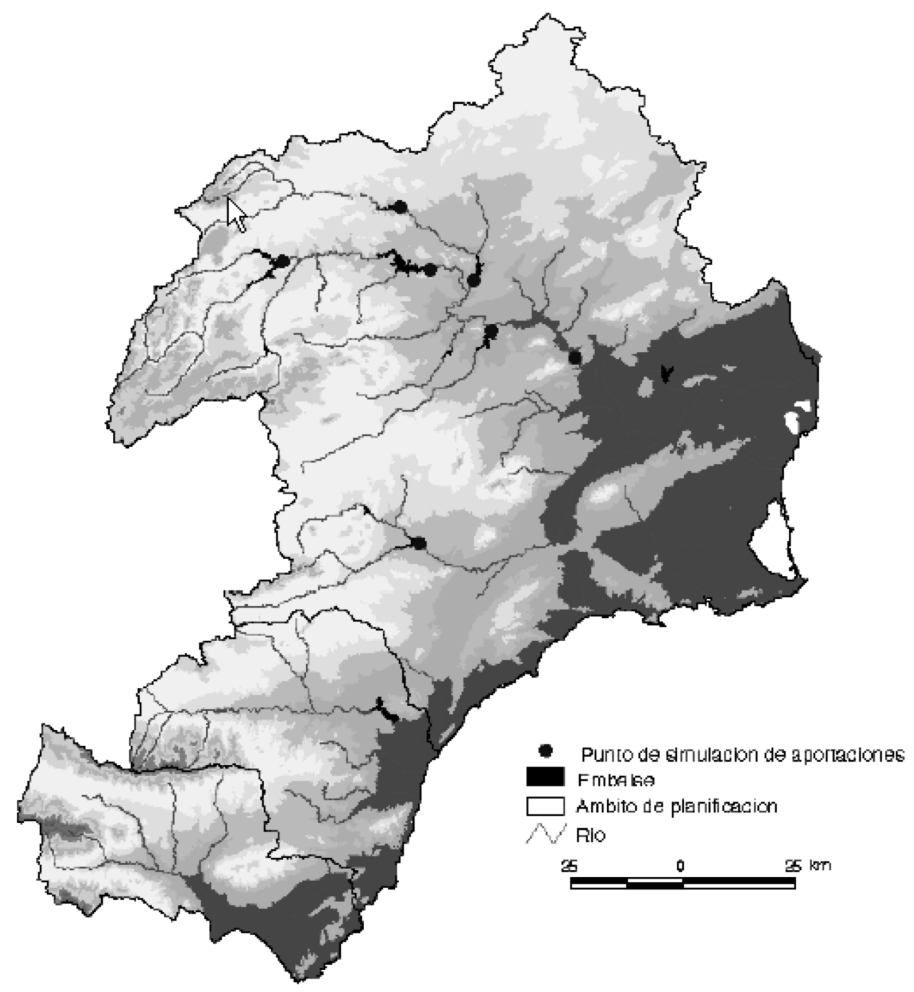

Figura 10. Puntos básicos de evaluación de recursos hídricos en la Cuenca del Segura (PHN).

Es por ello por lo que hemos procedido a evaluar los recursos hídricos de la Región de Murcia mediante la modelización de los elementos climáticos básicos que intervienen en el ciclo del agua. Estos elementos han sido los registros de las precipitaciones $(\mathrm{P})$ y el cálculo de las evapotranspiraciones potenciales (ETP) y reales (ETR). Elementos de cuyas interacciones surge el concepto básico de la escorrentía total o recursos hídricos disponibles $(\mathrm{E})$ $\mathrm{o}$ aportaciones en régimen anual $(\mathrm{A})$ :

$$
\mathrm{E}(\mathrm{A})=\mathrm{P}-\mathrm{ETR}
$$

En el conjunto de estas correlaciones, los valores de ETP son los que suscitan las mayores dificultades e incertidumbres de cálculo ya que la diversidad de métodos, teóricos y experimentales, conducen a magnitudes muy diversas en intensidad y régimen. En la presente estimación de la evapotranspiración potencial (ETP) se ha utilizado el método de Thornthwaite. Frente a todas las posibles incertidumbres, la fórmula de Thornthwaite ha sido debidamente validada mediante la comparación de sus magnitudes con los coeficientes de escorrentía de algunas estaciones de aforos situadas en cuencas de cabecera no afectadas en su régimen natural en los sistemas hidrográficos del Segura y del Júcar. Esta fórmula ha sido ponderada con la de Turc para las regiones más secas y con $\mathrm{P}<\mathrm{de} 400 \mathrm{~mm}$ (Almarza, 1984). 


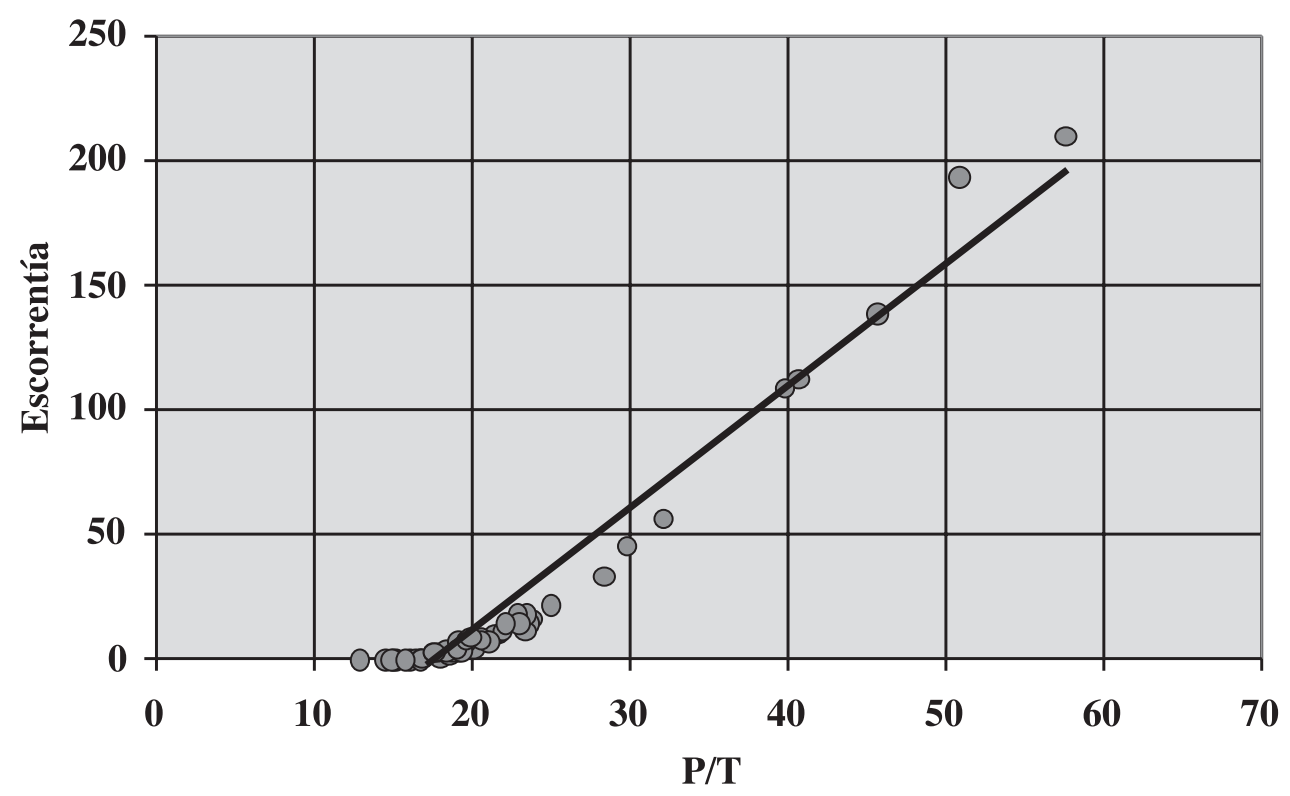

FIGURA 11. Relación entre la escorrentía regional y los valores de P y T.

No obstante, sobre una región con un régimen tan extremadamente irregular de las precipitaciones y de tan compleja configuración orográfica como la de Murcia, con sólo un 32 $\%$ sobre los 600 metros de altitud, las cantidades de agua disponibles no permiten siempre alcanzar el valor potencial de la ETP. En consecuencia, el valor de la escorrentía total viene mejor determinado a través del concepto de la ETR o evapotranspiración real. Un concepto cuyo valor ha podido ser calculado merced a la buena y densa red de observatorios meteorológicos regionales. De este modo, y definida la E, escorrentía o aportación total, como la diferencia entre la Precipitación y la ETR, la función que relaciona estas magnitudes es:

$$
\mathrm{E}=\mathrm{P}-\mathrm{ETR}
$$

Consecuentemente y dadas las estrechas relaciones existentes entre los elementos climáticos que intervienen en el ciclo hidrológico natural, ha sido posible establecer la función de ajuste que vincula las escorrentías totales con las precipitaciones. Los resultados obtenidos sobre los treinta observatorios de mayor longitud de observación y homogeneidad de datos de la Comunidad de Murcia han dado un ajuste bastante aceptable (figura 11).

\subsection{El escenario climático actual sobre la Región de Murcia y su situación hidrológica}

Ha sido representado mediante la cartografía de los principales elementos climáticos que intervienen en el ciclo hidrológico. De este modo, la cartografía climatológica actual de la Región de Murcia está basada en los valores de la precipitación (P) (figura 12) y temperatura (T) (figura 13) extraídos de las series climáticas de la red de observatorios regionales del INM y de la Confederación Hidrográfica del Segura. 


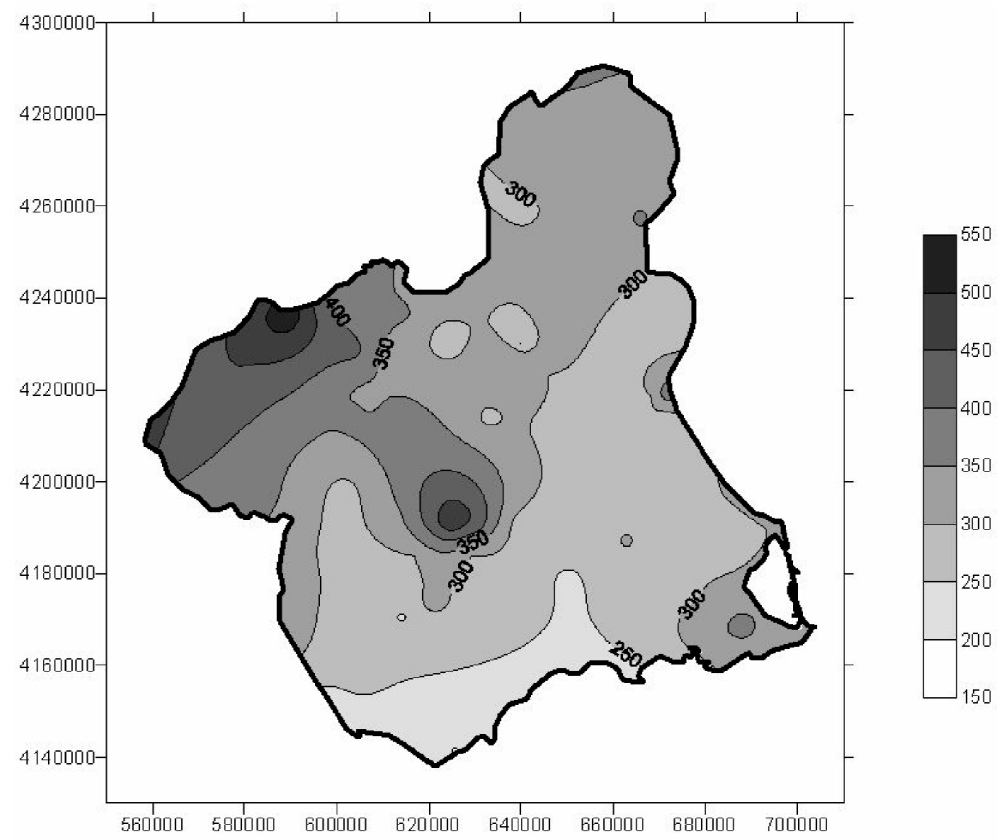

FIGURA 12. Precipitaciones medias sobre la Región de Murcia (1950-2000).

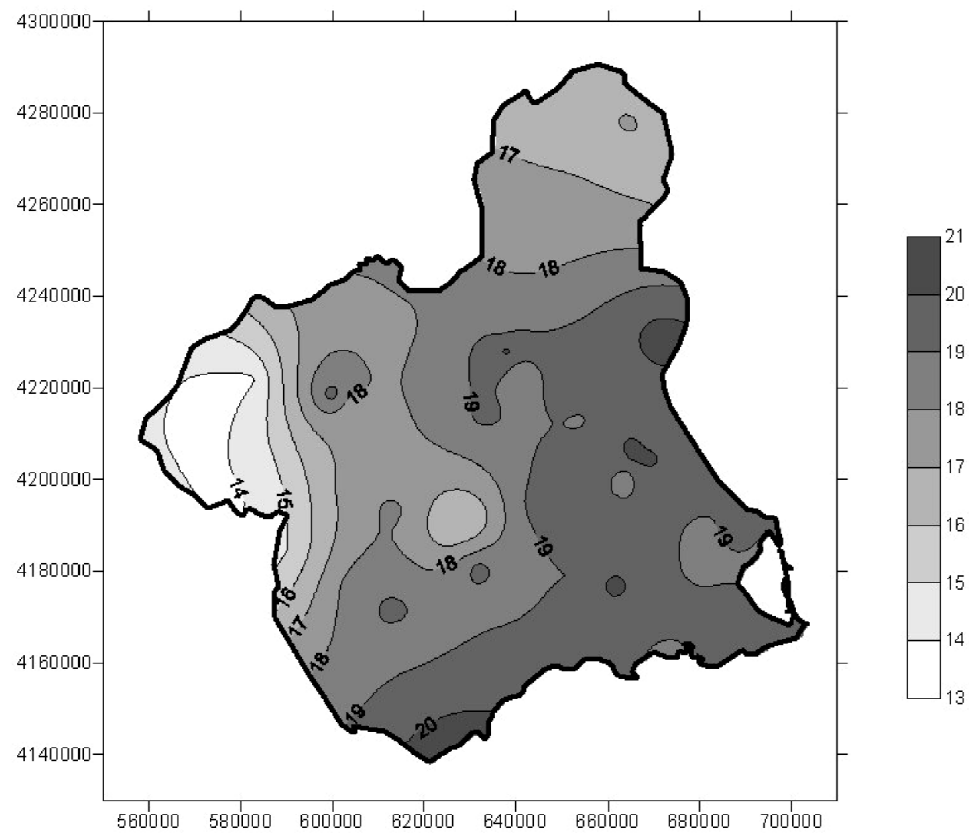

FIGURA 13. Temperaturas medias anuales sobre la Región de Murcia. 


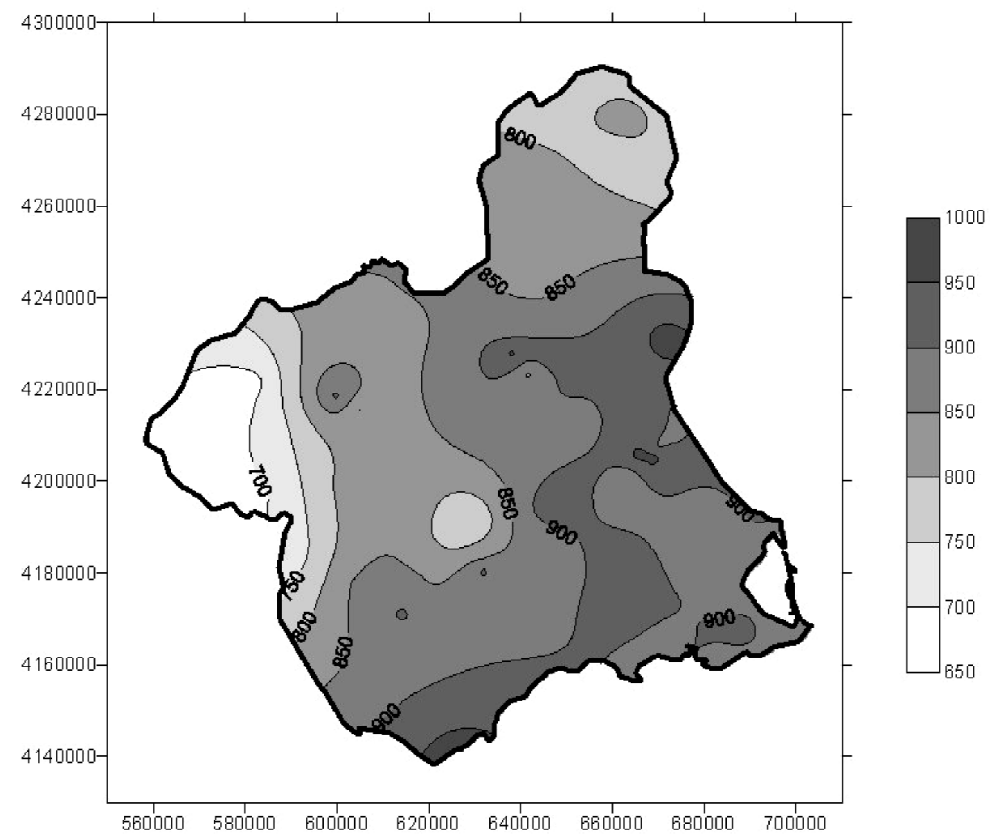

FIGURA 14. Evapotranspiraciones potenciales medias (ETP) sobre la Región de Murcia (1950-2000).

El valor de la ETP, como ya se ha dicho, ha sido calculado en función del método de Thornthwaite (figura 14). Estos datos han servido, de acuerdo a la metodología expuesta, para elaborar el mapa de la escorrentía total (E) o recursos hídricos disponibles en régimen natural sobre la Región de Murcia.

A partir de estos mapas climáticos de la situación actual se ha calculado el valor de las evapotranspiraciones reales (ETR). El procedimiento utilizado para este cálculo ha sido la elaboración de las correspondientes fichas hídricas de cada una de las veinte mejores estaciones termopluviométricas. Consecuentemente la relación mensual entre las variables de la precipitación y la ETP paralelamente a los procesos de reserva de agua en el suelo ha permitido calcular adecuadamente la ETR (figura 15).

En este cálculo, la escorrentía correspondiente a valores de precipitación inferiores a $400 \mathrm{~mm}$ ha sido ajustada en función del método de Thornthwaite y de Turc. A partir de esta curva de ajuste ha sido calculada la escorrentía total y proyectada cartográficamente mediante MDT. El modelo utilizado aquí ha sido de naturaleza teselar o raster de puntos georeferenciados distribuidos regularmente sobre el territorio. La interpolación de los valores calculados sobre el territorio sin observaciones ha sido el kriging con función de correlaciones espaciales aplicadas mediante el programa Surfer. Finalmente, la obtención de los valores promedios regionales ha sido calculada mediante la computación de matrices o álgebra de mapas efectuada a través del SIG-IDRISI. Tales valores son los que figuran en la base cartográfica elaborada como mapa de la escorrentía total media anual en mm sobre el territorio de la Comunidad Autónoma de Murcia (figura 16).

Este mapa viene a mostrar la gran limitación de los recursos hídricos disponibles sobre la región. En su mayor parte el territorio está por debajo de los $10 \mathrm{~mm}$ de escorrentía media 


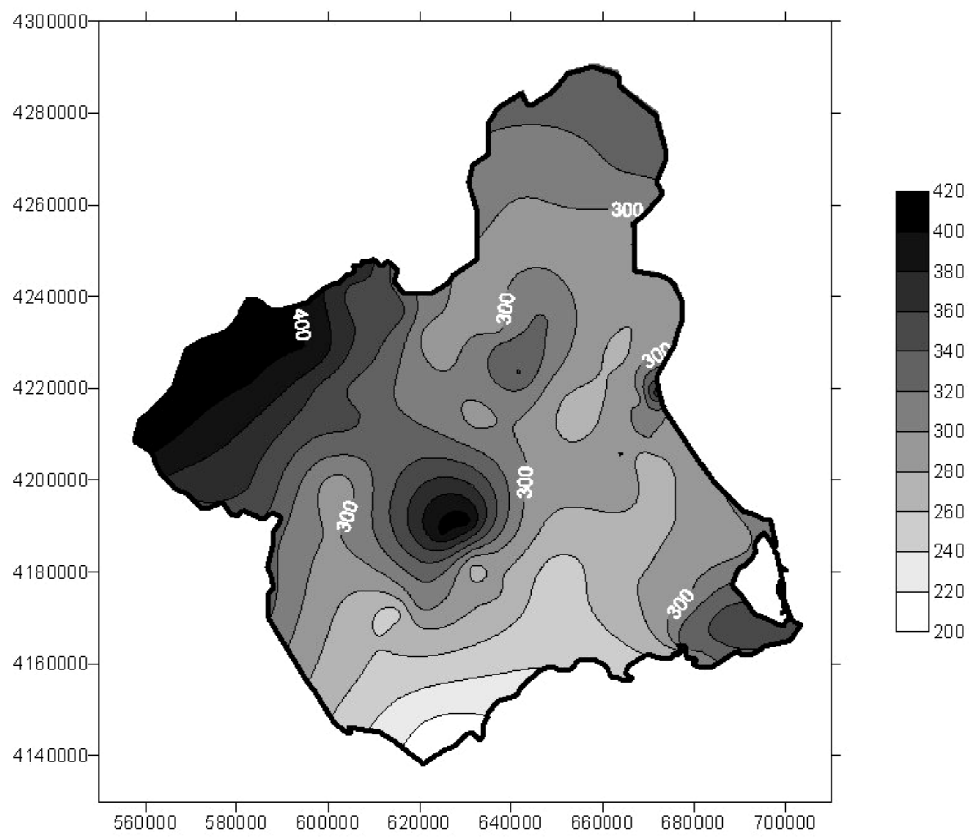

FiguRA 15. Mapa de la ETR (en mm) sobre la Comunidad de Murcia (1950-2000).

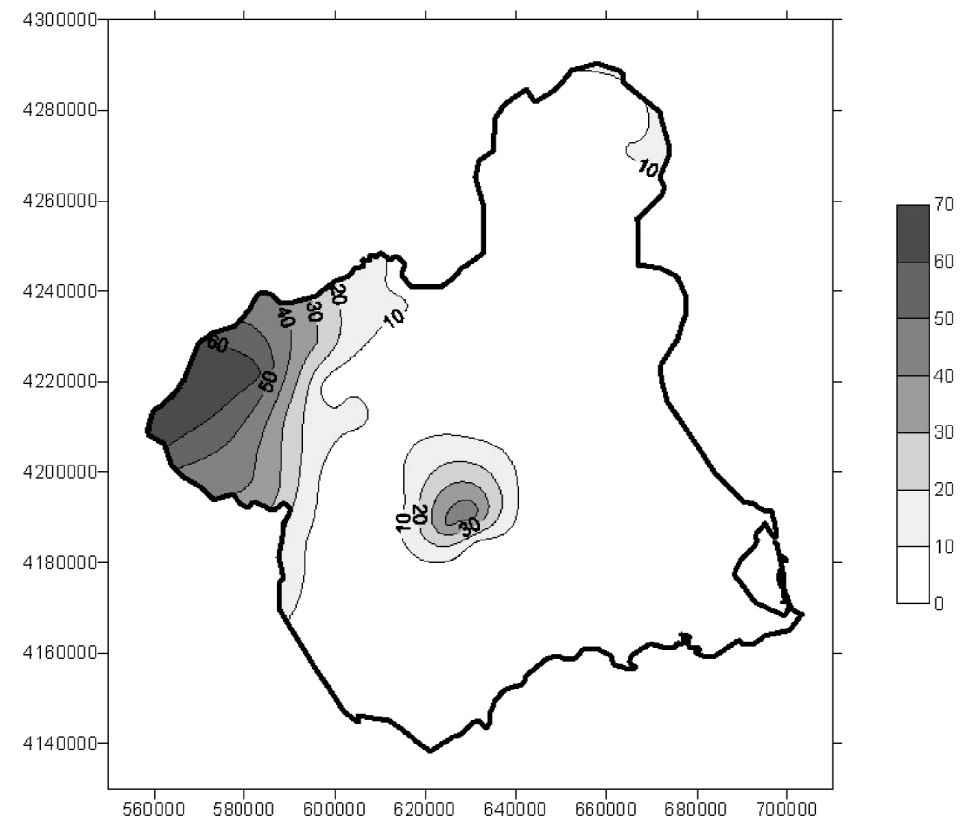

FiguRa 16. Escorrentías totales medias (mm) sobre la Región de Murcia (1950-2000). 
anual y tan sólo en dos áreas se sobrepasa claramente. La primera está situada sobre las Sierras Occidentales, cabeceras de los ríos Segura y Mundo, mientras que la segunda corresponde al macizo de Sierra Espuña. La escorrentía media anual se sitúa sobre los $8.61 \mathrm{~mm}$, valor muy inferior a los $212 \mathrm{~mm}$ de media española. Ello es consecuencia de los bajos coeficientes de escorrentía regionales, $11 \%$ frente al $60 \%$ que se obtienen en las cuencas septentrionales de España. Globalmente, y sobre los $11.314 \mathrm{Km}^{2}$ del territorio murciano, suponen tan sólo $97.5 \mathrm{Hm}^{3}$ anuales de recursos hídricos propios.

A partir de estos datos hemos procedido a estimar cual sería el impacto que sobre los recursos hídricos de la Región de Murcia tendría la variación termopluviométrica prevista en los dos escenarios climáticos desarrollados. Para esta estimación se ha utilizado la función regional que relaciona la escorrentía anual según métodos de Thornthwaite y Turc con el coeficiente $\mathrm{P} / \mathrm{T}$. Como hemos visto anteriormente en la figura 11 el ajuste con un valor de $\mathrm{R}^{2}$ superior al 0.95 es muy aceptable. En cualquier caso, los resultados mostraban una mejor validación con la realidad a los obtenidos mediante la ley de Budiko (1961) utilizada en el Plan Hidrológico Nacional.

\subsection{El escenario climático en el horizonte del año 2050, bajo el aumento térmico de $0.5^{\circ} \mathrm{C}$, y su impacto sobre la escorrentía total}

A medida que las condiciones climáticas se van alterando su principal repercusión se produce sobre el ciclo hidrológico. En este sentido, el mapa de la figura 17 representa las magnitudes de escorrentía que cubrirían el territorio de Murcia bajo condiciones de un aumento térmico de medio grado centígrado y una sostenibilidad de las precipitaciones en los valores medios actuales. Este impacto, tal y como muestra la figura 17, equivale a una

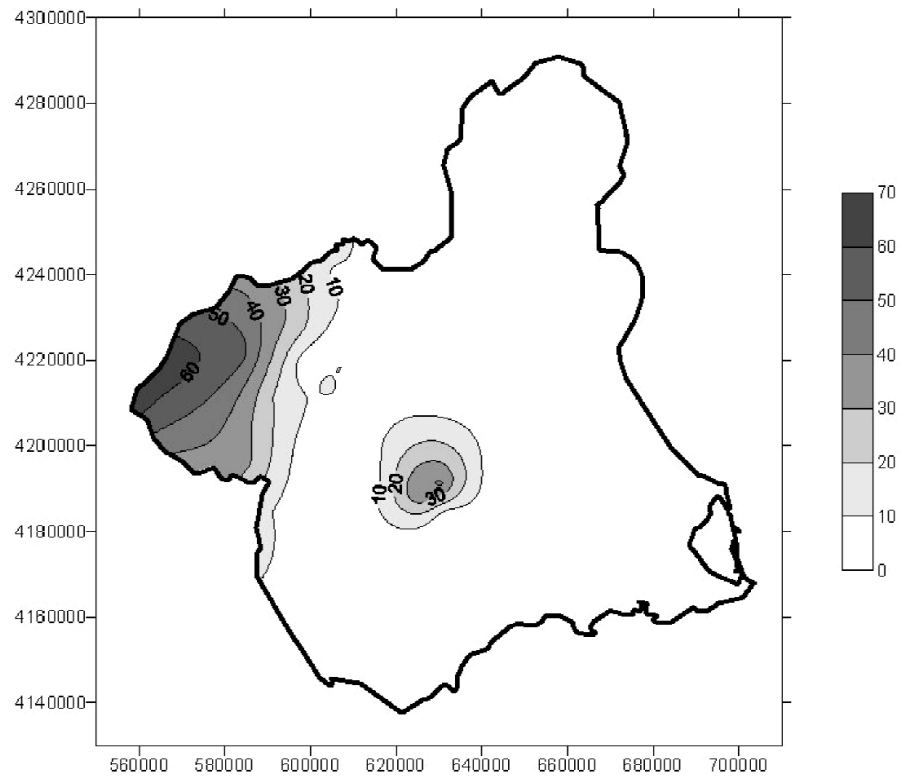

FIGURA 17. Mapa de escorrentías previsto en el horizonte del año 2050 y en el escenario climático II. 
reducción del $15 \%$ en los recursos hídricos y paralelamente reflejan el aumento de las evapotranspiraciones en todas las ocasiones en que tal escenario térmico se produce. La escorrentía media anual se sitúa en $7.41 \mathrm{~mm}$.

\subsection{El escenario climático en el horizonte del año 2050, bajo el aumento térmico de $0.5^{\circ} \mathrm{C}$, y una reducción del $5 \%$ en el volumen de sus precipitaciones}

Viene a determinar el impacto más severo sobre el ciclo hidrológico y los recursos hídricos de la Región de Murcia. El 85 \% del territorio de la región estaría por debajo de los 10 $\mathrm{mm}$ de escorrentía total anual. Regionalmente ello supondría una reducción en los recursos hídricos propios de hasta un $40 \%$ de los actuales valores que quedarían reducidos a tan sólo $5 \mathrm{~mm}$ (figura 18).

\section{Conclusiones}

El objetivo del presente estudio ha sido el de analizar y proyectar los impactos que un cambio climático podría tener sobre el territorio de la Comunidad de Murcia. Un objetivo de gran trascendencia a tenor de las actuales predicciones que los modelos desarrollados establecen sobre nuestra región. Según estos modelos, a lo largo del siglo XXI, se podría asistir a una sensible readaptación de su escenario biogeográfico. Éste no sólo se vería afectado por el aumento general de las temperaturas sino que muy especialmente, y dado que en la Naturaleza todo aparece gobernado por lo térmico, asistiría a una sensible reducción de sus recursos hídricos tanto por la disminución de precipitaciones como por el aumento de las evapotranspiraciones.

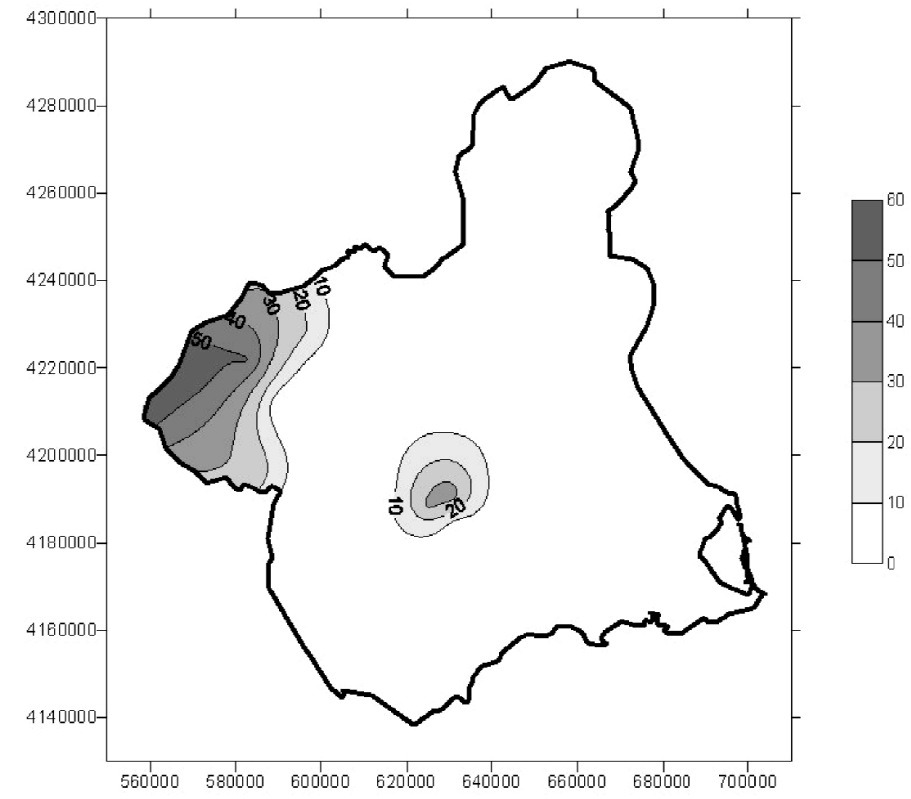

FIGURA 18. Mapa de escorrentías previsto en el horizonte del año 2050 y en el escenario climático III. 
En consecuencia, nuestro estudio ha tratado inicialmente de analizar una cuestión básica ¿Se están cumpliendo ya esos escenarios previstos por los modelos sobre la región mediterránea española? Ésta es propiamente la cuestión o reto científico que tiene ante sí la Climatología mediterránea: ¿Están ascendiendo las temperaturas?, ¿Están disminuyendo las precipitaciones de la región mediterránea? Ciertamente que hoy no es posible responder de modo enteramente satisfactorio a estas inquietantes cuestiones. Nuestro actual conocimiento de la dinámica atmosférica general y regional es insuficiente para afrontar con garantía este reto científico. Sin embargo, y siempre desde un punto de vista climatológico, es lícito que a partir de nuestro actual conocimiento del cómo evoluciona el clima mediterráneo, podamos interrogarnos por el hacia dónde se dirige. Es decir, el remontarnos desde el terminus ad quo actual hasta el terminus ad quem futuro.

A tenor de los análisis efectuados, nuestras conclusiones sobre las consecuencias de un posible cambio climático sobre la Región de Murcia no descartan la posibilidad de un sostenimiento climático dentro de la variabilidad natural de nuestro clima. No obstante, con las incertidumbres expresadas y ante la gran sensibilidad que los recursos hídricos muestran frente a las variaciones climáticas, hemos realizado una proyección sobre las tendencias termopluviométricas más severas observadas durante el siglo XX. En el horizonte más adverso de estos escenarios, el impacto sobre los recursos hídricos podría revestir una notable gravedad al convertir el territorio de Murcia en una auténtica zona de clima desértico con reducción de hasta un $40 \%$ de las actuales escorrentías. En síntesis, la gran sensibilidad del sistema hidrológico a los posibles efectos de un cambio climático, muestra el enorme interés que adquiere la adopción de las políticas de ahorro y gestión ante las demandas futuras.

\section{Bibliografía}

ALMARZA, C. (1984). Fichas hídricas normalizadas y otros parámetros hidrometeorológicos, INM, 3 vols.

ALEXANDERSSON, H. and MOBERG, A. (1997). «Homogenization of swedish temperature data. Part I: Homogeneity test for linear trends». International Journal of Climatology, 17, pp. 25-34.

AYALA-CARCEDO, J. e IGLESIAS, A. (2000). Impactos del posible cambio climático sobre los recursos hídricos, el diseño y la planificación hidrológica en la España Penínsular, BBVA, Servicio de estudios.

CERDÁ CERDÁ, A. (2003). «Prólogo», en Seguimiento y evaluación de los efectos sobre el medio natural de la sequía y los procesos erosivos en la Región de Murcia, Consejería de Agricultura, Murcia.

CONFEDERACIÓN HIDROGRÁFICA DEL SEGURA (1999). Plan Hidrológico de Cuenca del Segura.

IPCC (1992). «Climate Change: The Supplementary Report to the IPCC Scientific Assessment», J. T. HOUGHTON, B. A. CALLENDER and S. K. VARNEY (eds.). Cambridge University Press, Cambridge, UK, 198 pp.

IPCC Working Group I Third Assessment Report (2001). Climate Change 2001: The Scientific Basis. Draft version in http://www.gcrio.org/online.html. 18 pp.

IPCC (1996). «Climate Change 1995: The Science of Climate Change: Contribution of Working Group I to the Second Assessment Report of the Intergovernmental Panel on Climate Change». J. J. HOUGHTON, L.G. MEIRO FILHO, B. A. CALLANDER, N. HARRIS, A. KATTENBERG AND K. MASKELL (eds). Cambridge University Press, Cambridge, UK, 584 pp. 
IPCC Working Group I Third Assessment Report (2001). Climate Change 2001: The Scientific Basis. Draft version in http://www.gcrio.org/online.html. 18 pp.

JONES, P. D. (1996). «1995-The warmest year on record (so far)». Draft Press Realease for january 1996. Web Homepage of the Climatic Research Unit, University of East Anglia, Norwich, England.

MIMAM (2000). El libro Blanco del Agua en España, Madrid, Ministerio de Medio Ambiente, $637 \mathrm{pp}$.

MIMAM (2001). Plan Hidrológico Nacional, Madrid, Ministerio de Medio Ambiente, 5 vols.

PARRY et al. (1999) Informe ACACIA, (Projet: A Cocertated Action Towards a Comprensive Climate Impacts and Adaptations Assessment for the European Union).

QUEREDA SALA, J., GIL OLCINA, A., PÉREZ CUEVA, A., OLCINA CANTOS, J., RICO AMORÓS, A. and MONTÓN CHIVA, E. (2000). «Climatic warming in the Spanish Mediterranean: Natural Trend or Urban effect», Climatic Change, 46, 4. Univ. de Standford, Kluwer Academic.

THORNTHWAITE, C.W. (1948). Micrometeorology of the surface layer of the atmosphere. Public. Climat. 1 bis, 5.

THORNTHWAITE, C.W. (1948). «An approach toward a rational classification of climate». Geographical Review: 55-94.

THORNTHWAITE, C.W. and MATHER, J.R. (1955). The water balance. Publications in Climatology 8 (1).Laboratory of Climatology. Centerton, N.J.

TURC, L. (1961). «Evaluation des besoins en eau d'irrigation, evapotranspiration potencielle», Ann. Agron., pp.13-49. 